\title{
Vom Leben erzählen: Ein Konferenzbericht zu szenischem Lernen und literarischem Verstehen
}

\section{Tom Klimant}

\begin{abstract}
Im Rahmen des Bayreuther Germanistentages 2016 widmete sich das Panel ,Vom Leben erzählen: Literaturdidaktische Perspektiven auf Supplemente alltäglichen Erzählens' zunächst praktisch im Rahmen eines Workshops und dann theoretisch im Kontext einer Ideenwerkstatt der Einbindung lebensweltlicher Bezüge Lernender aus Schule und Hochschule in literarische und poetische Vorstehensprozesse vor dem Hintergrund theatralen Lernens. Nachfolgend soll über zentrale Aspekte und Fragestellungen, Abläufe und Übungsformen dieses Panels berichtet werden.
\end{abstract}

\section{Lebensweltbezüge im Literaturunterricht}

Schulunterricht als artifizieller Raum ermöglicht Lehr-Lern-Konstellationen, die Laborsituationen ähneln und besondere Schutzräume generieren, zugleich fordert er die Lehrenden heraus, die Lebensweltbezüge der Schülerinnen und Schüler nachvollziehbar und erlebbar in das Geschehen einzubinden und so die Membran zwischen Lebenswirklichkeit und Unterricht für Lernende durchlässig zu gestalten. Wolfgang Klafki profilierte in seinen bildungstheoretischen Studien auch diesen Aspekt unter dem Blickwinkel der zu legitimierenden Auswahl eines Unterrichtsgegenstandes (cf. Klafki 2007: 270ff.). In der Praxis erweist sich diese wichtige und vielseits gestützte Forderung nicht immer und als leicht realisierbar. Mit dem Panel begaben sich Lehrende aus Hochschule und Schule im Rahmen des Deutschen Germanistentages in Bayreuth 2016 gemeinsam und in Anlehnung an Konzepte des ,artistic research' bzw. ,practice as research' auf die Suche nach Möglichkeiten theatraler Erlebens- und darauf basierender Lehr/-Lernräume im Literaturunterricht. Das Panel richtete sich an theoretisch und praktisch Interessierte, die ihre Auseinandersetzung mit literatur- und theaterdidaktischen und mit theaterpädagogischen Konzepten vor allem im schulischen, aber auch im universitären Literaturunterricht erweitern und vertiefen wollten.

Zunächst wurde ein Workshop mit allen Teilnehmenden durchgeführt. Ziel war es, Möglichkeiten der Implementierung alltäglicher Erlebens- und Erfahrenswelten von Schülerinnen und Schülern in den Unterricht vermittels 
theatraler Lehr-Lernrprozesse praktisch zu erproben und das im Theaterspiel Produzierte wiederum literarischen bzw. poetischen Verstehensprozessen im Literaturunterricht zuzuführen. Im Rahmen eines daran anschließenden World Cafés wurden mit Blick auf eine Vertiefung der Arbeit über das Panel hinaus Leitfragen gemeinsam aufgefächert und Forschungsperspektiven entwickelt.

\section{Theatrales Lernen und literarisches Verstehen}

\subsection{Theatrale Haltungen erzählen von einer beredten Lebenswelt}

Erzählen als Erwerb eines Bündels vielfältiger produktiver narratologischer Kompetenzen findet in der didaktischen Forschung ebenso Berücksichtigung wie die Rezeption medial unterschiedlich repräsentierter ästhetischer Formen des Erzählens (cf. Claussen 2004; Leubner und Saupe 2009; Dannerer 2012). Größere mündliche, erfahrungsbezogene Erzählzusammenhänge werden vor allem in der Elementar- und Primarstufe und in den frühen Jahrgängen der Sekundarstufe I berücksichtigt.

Darüber hinaus spielt das Erzählen oft eine nachgeordnete Rolle; dabei ist es mit Blick auf literarische und poetische Verstehensleistungen (cf. Abraham 2010; Wintersteiner 2010) besonders in der Sekundarstufe II mit Kompetenzen und Fähigkeiten verbunden, für deren Förderung es sich als günstig erweist, theatrale produktive Lernvorgänge und theatrale ästhetische Erlebens- und Erfahrensprozesse systematisch einzubeziehen.

Dieses Panel setzte nun dort an, wo Erzählen im Alltag stattfindet, außerhalb der Schule, im familiären Umfeld, in der Peergroup, in den Medienangeboten oder im öffentlichen Raum. Dort, wo Menschen beobachtbar sind. Deren körperliche Haltungen, Gesten und Gebärden können Anlass dafür sein, Schülerinnen und Schülern von ihrem unbekannten Gegenüber zu ,erzählen'. Fremdbegegnung, Aktivierung der individuellen Vorstellungskraft und - bezogen auf theatrale Lehr-Lern-Prozesse - Leiblichkeit im Kontext literarischen und poetischen Verstehens setzen nicht erst im Klassenraum an. Die Teilnehmenden wurden zunächst praktisch-produktorientiert mit einem theatralen methodischen Baukasten zur Einbindung solcher alltäglicher, beredter Gesten und Haltungen in den Literaturunterricht vertraut gemacht dies nicht im Sinne eines best-practice-Beispiels, sondern um eine gemeinsame, konkrete Erlebensgrundlage auch unter den Panelteilnehmenden mit Blick auf die daran anschließende Ideenwerkstatt anzubahnen.

\subsection{Alltägliches Erzählen}

Mit dem Titel ,Alltägliches Erzählen' verbinden sich, je nach unterschiedlich prononcierter Lesart, zwei thematische Ausrichtungen, die in einer Perspektive konvergieren: Alltägliches erzählen und alltägliches Erzählen.

(1) Alltägliches Erzählen: ,Am Anfang war die Geste' sagt Jürgen Belgrad im Rahmen des Symposion Deutschdidaktik 2016 mit Blick auf Michael Tomasellos 
Arbeit ,Die Ursprünge der menschlichen Kommunikation'. Ein Blick in die gegenwärtige Gestenforschung oder die anthropologische Bildungsforschung des Theaterspiels (cf. Liebau 2008) stützt die damit verbundene These nach einem Primat des Leiblich-Gestischen und, daran anknüpfend, auch der theatralen Haltung und des theatralen Gehens im Kontext kommunikativer Prozesse, in denen der Beobachtende etwas über sein Gegenüber erfährt oder zu erfahren meint. Das, was sich hier mitteilt, dient ihm oder ihr zur Orientierung bspw. an hypothetischen personalen Merkmalen zur raschen, d.h. auch heuristischen Einschätzung des Menschen. Anders als im Klassenraum gibt es hier noch keinen Dritten, keinen expliziten Zuschauenden. Das heißt nicht, hier gebe es kein Theater. Die Workshop-Akteure wurden zu Spielenden im öffentlichen Raum. Das sog. ,Abnehmen' alltäglicher Haltungen und deren Anverwandlung als theatrale Haltungen (cf. Ebert 1998: 84), das aus theaterpädagogischer und -didaktischer Sicht als fester methodischer Bestandteil theatraler Schaffensprozesse gilt (cf. bspw. Köhler 2016: 17), kann eine wichtige Schnittstelle zwischen Lebenswirklichkeit, theatralem Lernen und Literaturunterricht etablieren.

(2) Alltägliches Erzählen: Die Teilnehmenden setzten sich zunächst im geschützten Rahmen des ,Klassenraumes' theaterspielend mit Begriffen der theatralen Haltung und des theatralen Gehens auseinander, um in einem zweiten Schritt aus diesem Raum heraus zu treten und konkrete Gänge und Haltungen ,abzunehmen' und in die fingierte Unterrichtssituation $\mathrm{zu}$ implementieren. Drittens führte dies entsprechend zu einer Auseinandersetzung mit literarischen Figuren und zu einer Eröffnung literarischer bzw. poetischer Verstehensprozesse im Kontext theatralen Lernens und handlungs- und produktionsorientierten Literaturunterrichts. Die Anschlusskommunikation an das nachahmende und darstellende - und in diesem Sinne - mimetische (cf. Petersen 2000) Finden und Anverwandeln alltäglicher Haltungen wurde ebenfalls theaterspielend realisiert; dadurch konnten wertvolle ästhetische Erlebensqualitäten bewahrt werden. Josef König argumentiert, dass sich ästhetische Wirkung wieder nur adäquat, also sie daselbst aufnehmend und zur Geltung bringend, in ästhetischer Wirkung niederschlagen könne (cf. König 1974). Mediale Wechsel (etwa in die Sprache) schließt dies ein; entscheidend ist jedoch, dass der leibliche Schaffensraum des Theaterspiels dann in eine der Materialität der Sprache zugewandte und sprach-spielerische Kommunikation übergeht.

Durch die theatralen Lehr-Lern-Situationen war es den Teilnehmenden möglich, solche Momente des Alltags aufzuspüren, die je individuell als ,erzählend' wahrgenommen wurden. Aus Sicht der Theaterlehrperson konnte dadurch zugleich die berüchtigte Komplexitätsfalle der Binnendifferenzierung vermieden werden. Interesse und Neugierde sollten vor dem Hintergrund geweckter Spiellust (cf. Barz 1998: 51) und emotionaler Offenheit (cf. Jenisch 1984: 404) die Akteure einladen, sich den sie ansprechenden Haltungen und Gesten des Alltags mimetisch zuzuwenden, anzunähern und sie durch eigenes Spiel beredt werden zu lassen. 


\subsection{Theatraler Spielraum im Klassen- und Alltagsraum}

Der theatrale Spielraum wird über den Klassenraum hinaus in den Alltagsraum verlängert. Beobachtend, ergreifend und ergriffen, nacheifernd, markierend, simulierend, stilisierend, nachspürend, im umfassenden Sinne wahrnehmend und verkörpernd wird auch dort Theater gespielt, obgleich und gerade weil ausnahmsweise keine Zuschauenden (und vor allen Dingen keine Lehrpersonen) anwesend sind. Für alle Beteiligten ist transparent, dass das gefundene Material, dass die prozesshaften, letztlich singulären Schüler- und Schülerinnenprodukte, gewürdigt und weiteren Arbeitsschritten zugeführt werden. Eine zentrale Fragestellung dieser Workshop-Phase lautete entsprechend: Wie kann die Begegnung mit einer fremden, körperlichen und nur beobachtbaren Haltung dazu beitragen, diese als das Fremde im Eigenen im Sinne einer Perspektivübernahme zu entdecken und spielerisch (und eben nicht kognitiv) zu erkunden und es dann mit literarischen Figuren in Berührung zu bringen. Spiellust und ästhetisch-erlebende Anschlusskommunikation, Aufmerksamkeitsschulung und systematisch vermittelte und in ihren Wirkungszusammenhängen erlebte theatrale Werkzeuge sowie die Bereitschaft, sich auf Atmosphäre (cf. Böhme 1995) und Resonanz (cf. Rosa 2016: 246ff. und 331ff.), Konzentration und Durchlässigkeit für eigene und fremde Impulse einzulassen und einlassen zu können, erwiesen sich hier als wichtige Markierungen.

\section{Didaktische Potenziale der Spuren alltäglichen Erzählens im Literaturunterricht}

Den didaktischen Potenzialen solcher Spuren alltäglichen Erzählens wurde im Rahmen des Panels vor dem Hintergrund der Leitfrage nachgegangen, wie literarische und poetische Verstehensprozesse am Beispiel literarischer Figuren durch den theatralen gestalterischen Umgang mit Erscheinungsformen alltäglichen Erzählens gefördert werden können.

Theatraler Gang und theatrale Haltung verstanden sich hier als Keimzellen dessen, worin sich das ,alltägliche Erzählen' realisiert und verdichtet. Vielleicht weckt bspw. die Haltung des auf einer Parkbank sitzenden und die Spatzen beobachtenden Mannes Assoziationen an einen Gregor Samsa, der im fortgeschrittenen Alter auf das Erlebte zurückschaut. Haltung und Gang sind zentrale Konzepte der praktischen Theaterarbeit, die nicht allein kognitiv beschrieben und erschlossen werden können, sondern die auch und vor allem praktisch erlebt und erfahren werden müssen - und weit mehr und intensiver als dies im Rahmen eines Workshops möglich ist; dennoch war eine Annäherung an eine gemeinsame Erlebensbasis auch in dieser heterogenen Gruppe Lehrender aus Schule und Hochschule möglich. 


\subsection{Didaktische Reflexionen exemplarischer Übungen und Etüden}

Nach körperlichen Lockerungsübungen unterstützten auf den eigenen und fremden Leib bezogene Wahrnehmungs- und Isolationsübungen die Darstellenden darin, einzelne Partien des Körpers, insbesondere Gelenke und Extremitäten und die damit verbundenen Freiheitsgrade, hinsichtlich nonverbaler und proxemischer kommunikativer Prozesse im Raum, mit dem Partner und in der Gruppe spielerisch zu erforschen. In der daran anschließenden Übungsphase nahmen die Beteiligten körperliche, wiederholbare Formeinheiten (shapes und patterns) einander unmittelbar in Partnerarbeit ab - dabei wurde das schauspielpädagogische ,Zug-um-Zug'-Prinzip insofern modifiziert, als die Bewertungsphase möglichst kurz gehalten und so die Unmittelbarkeit der physischen Reaktion (response) im Schaffensvorgang betont wurde. Der Wechsel zwischen der leiblichen Handlung (action) von A, der B zuschaut, und umgekehrt, fand möglichst direkt statt. Damit verbunden tauchten (und tauchen immer wieder typischerweise) Fragen infolge wichtiger introspektiver Beobachtungen auf, etwa: ,Ich war unzufrieden mit meiner Einfallslosigkeit und wusste nicht mehr, was ich dann tun konnte'. Andere nahmen diese Aufgabenstellung und den damit verbundenen Spielraum als ,befreiend' wahr. Deutlich wird das Wider- und Zusammenspiel von ,innerem Kritiker' und Zensor einerseits und der Möglichkeit, den Körper näherungsweise unvoreingenommen auf das Wahrgenommene reagieren zu lassen, andererseits. Sich des eigenen Erlebens bewusst zu werden und es zugleich Teil der physischen Antwort sein zu lassen und sein lassen zu dürfen (being in the moment), auch im Falle weniger gewünschter Gefühle oder Vorstellungen von der eigenen Außenwirkung, stellte - neben zahlreichen weiteren bemerkenswerten Äußerungen - einen zentralen Aspekt dar. Die Frage, ,wer' das ist, der hier reagiert, bot im Nachgang der Praxis spannende Anschlussstellen für die interdisziplinäre Erforschung eines bewusstseinsphilosophisch-kognitionswissenschaftlichen Subjektverständnisses der produktionsorientierten Theaterdidaktik jenseits essentialistischer Ansätze. In den folgenden Arbeitsphasen wurde unter anderem das Finden theatraler Gänge und Haltungen über schillernde Verben zu Gangarten im erweiterten Sinne (tänzeln, flanieren, taumeln usw.) und vermittels idiomatischer Redewendungen rund um das Thema ,Gehen' (,über den Jordan gehen', ,auf Tuchfühlung gehen', ,auf leisen Sohlen kommen', ,den Weg des geringsten Widerstandes gehen' usw.) erweitert. Zunehmend wurden Anreize geschaffen, Aspekte individuellen, körperlichen Ausdrucks möglichst spannungs- und wertungsfrei, konzentriert und interessiert (über Fragen des Atems, der zuvor etablierten Freiheitsgrade und Immobilitäten bzw. eingeschränkten Mobilität sowie der sie begleitenden Empfindungen bspw.) zu vertiefen, um über weitere Etappen hinweg einen, eigenen' Gang zu finden und fremde Gänge abzunehmen. Innere Narrative und Vorstellungen, Empfindungen und Assoziationen bildeten dabei wesentliche Ankerpunkte der Wahrnehmung eigenen Wahrnehmens; so arbeiteten die Teilnehmenden auch mit der Übertragung innerer Bilder infolge abgenommener Gänge auf eigene theatrale Gänge - stets mit dem Ziel verbunden, die Korrespondenz innerer und äußerer Haltung zu vergegenwärtigen: Ein 
Mensch nimmt eine innere Haltung ein und zeigt eine äußerlich wahrnehmbare körperliche Haltung.

Mit diesem Fundus eroberten die Teilnehmenden in einer zweiten Arbeitsphase den ,öffentlichen Raum', um dort vorzufindende Haltungen als eigene theatrale Haltung anverwandelnd aufzunehmen. Sie wurden im Theaterraum dann in kleinen etüdischen Formen spielerisch in Szene gesetzt, betrachtet und kontrastiert - im Sinne einer Anschlusskommunikation, die den ästhetischen Erlebensprozess möglichst fortsetzt. In der nun letzten Phase überflogen die Teilnehmenden kurze literarische Textpassagen. Angeboten wurden Absätze aus aktueller KJL und im Schulkontext kanonisierter Literatur. Eine im weitesten Sinne thematische Fokussierung des Unterrichts kann über die Stoffauswahl leicht hergestellt werden. Die Spielenden wählten nach Prozessen gezielt flüchtigen, peripheren und die Textur einbeziehenden Probierens einen auf wenige Worte verdichteten Textauszug, der sich ihrer vorgefundenen Haltung besonders anbot - damit war nicht unbedingt eine Passgenauigkeit im Sinne von Kongruenz gemeint und angestrebt. Vielmehr wurden Brüchigkeit, Widerständigkeit und Irritation durch Anleitung spielerisch in den Prozess systematisch eingebunden, um hier möglichst von Stereotypen und Verdoppelungen und letztlich Begrenzungen der theatralen Haltungen abzusehen. Leitend waren Fragen wie diese: ,Was möchte diese Figur verbergen? Was soll nicht gesehen werden, wird aber doch, ganz unbemerkt von ihr, sichtbar'? Was bleibt Dir rätselhaft? Was ist bloß ahnbar? Was weißt Du noch nicht von Deiner Figur?'. Mit Blick auf theatrale Schaffensprozesse werden Widerstände und Umwege als Chance zur Freisetzung besonderer Produktivität erachtet (cf. bspw. Sack 2011: 97; spezifisch für den Literaturunterricht Klimant 2016: 139ff.).

\subsection{Forschungsperspektiven}

$\mathrm{Zu}$ den Bereichen Genderperformance (Wiebke Dannecker), kulturreflexives Lernen (Tobias Akira Schickhaus), postmodernes Erzählen (Stefan Hofer), ,Experten des Alltags' (Bianka Zeitler), Anschlusskommunikation (Daniela Nägel und Anne Steiner) und Identitätsorientierung im Literaturunterricht (Julia Kuntz) wurden im Rahmen einer Ideenwerkstatt Forschungsfelder entfaltet und mit Blick auf Gegenstand und Ausrichtung der jeweils vertieften Aspekte mit diesen Fragen verbunden:

Stellen theatrale ästhetische Erfahrungen und Kompetenzorientierung einen Widerspruch und/oder eine Bereicherung mit Blick auf theatrale LehrLernprozesse dar?

Wie ist den Herausforderungen der Dokumentation und Bewertbarkeit solcher erlebens- und/oder erfahrungsbezogener Lernprozesse angemessen zu begegnen?

Welche Anforderungen ergeben sich an die theaterlehrende Deutschlehrerin und den theaterlehrenden Deutschlehrer?

In welcher Weise sind bestehende Methodenvielfalt und didaktische Ziele weiterhin zu differenzieren und zu profilieren? 
Welche Wirksamkeit entfalten Aufgabenstellungen und Impulse und inwieweit lassen sich diese für theatrale Lehr-Lern-Situationen systematisieren und konkretisieren?

Welche metakognitiven Prozesskompetenzen spielen eine zentrale Rolle im Kontext theatralen Lernens im Literaturunterricht?

Auf welche Weise lassen sich die skizzierten theatralen Lernphasen in Schreibimpulse und prozessorientiertes Schreiben überführen?

Welche Verbindungen ergeben sich zum Vortragen oder mündlichem Erzählen im Unterricht?

\section{Bibliografie}

Abraham, Ulf (2010): P/poetisches V/verstehen. Zur Eingemeidung einer anthropologischen Erfahrung in den kompetenzorientierten Deutschunterricht. In: Winkler, Iris; Masanek, Nicole \& Abraham, Ulf (Hrsg.): Poetisches Verstehen. Literaturdidaktische Positionen - empirische Forschung - Projekte aus dem Deutschunterricht. Baltmannsweiler: Schneider, 9-23

Barz, André (1998): Darstellendes Spiel. Berlin: Volk und Wissen

Böhne, Gernot (1995): Atmosphäre. Essays zur neuen Ästhetik. Frankfurt a.M.: Edition suhrkamp

Claussen, Claus \& Merkelbach, Valentin (Hrsg.) (2004): Erzählwerkstatt. Mündliches Erzählen. Braunschweig: Westermann

Dannerer, Monika (2012): Narrative Fähigkeiten und Individualität. Mündlicher und schriftlicher Erzählerwerb im Längsschnitt von der 5. bis zur 12. Schulstufe. Tübingen: Stauffenburg Verlag Brigitte Narr

Ebert, Gerhard (1998): Lernen zu improvisieren. In: Ebert, Gerhard \& Penka, Rudolf (Hrsg.): Schauspielen. Handbuch der Schauspieler-Ausbildung (4., überarb. und erg. Aufl.). Berlin: Henschel

Jenisch, Jakob (1984): Ausdrucksmöglichkeiten, Identifikationen und pädagogische Erfahrungen bei Theaterspielen. In: Kreuzer, Karl Josef (Hrsg.): Handbuch der Spielpädagogik. Band. 3. Das Spiel als Erfahrungsraum und Medium. Düsseldorf: Schwann, 397-417

Klafki, Wolfgang (2007): Neue Studien zur Bildungstheorie und Didaktik. Zeitgemäße Allgemeinbildung und kritisch konstruktive Didaktik (6., neu ausgestattete Aufl.). Basel und Weinheim: Beltz

Klimant, Tom (2016): Grenzverschiebungen theatralen Lernens. Zur Implementierbarkeit theatraler ästhetischer Spielräume in den Literaturunterricht. In: Steiner, Anne \& Radvan, Florian (Hrsg.): Theaterdidaktische Perspektiven auf Normen und Normbrüche im Drama und auf der Bühne. Baltmannsweiler: Schneider, 131-161 
Köhler, Eberhard (2016): Schauspielgrundlagen und Grundlagen der Regie. In: Hentschel, Ulrike (Hrsg.): Theater lehren. Didaktik probieren. Strasburg (Uckermark): Schibri-Verlag, 14-44

König, Josef (1974): Die Natur der ästhetischen Wirkung. In: Dehn, Wilhelm (Hrsg.): Ästhetische Erfahrung und literarisches Lernen. Frankfurt a.M.: Fischer, 63-80

Leubner, Martin \& Saupe, Anja (2009): Erzählungen in Literatur und Medien und ihre Didaktik. Baltmannsweiler: Schneider

Liebau, Eckart (2008): Was Schultheater für die Schüler und die Schule leistet. Dimensionen theatraler Bildung. In: Jurké, Volker; Linck, Dieter \& Reiss, Joachim (Hrsg.): Zukunft Schultheater. Das Fach Theater in der Bildungsdebatte. Hamburg: Edition Körber-Stiftung , 19-26

Petersen, Jürgen H. (2000): Mimesis - Imitatio - Nachahmung. Eine Geschichte der europäischen Poetik. München: UTB

Rosa, Hartmut (2016): Resonanz. Eine Soziologie der Weltbeziehung (4. Aufl.). Berlin: Suhrkamp

Sack, Mira (2011): Spielend denken. Theaterpädagogische Zugänge zur Dramaturgie des Probens. Bielefeld: Transcript

Wintersteiner, Werner: Wir sind, was wir tun. Poetisches Verstehen als fachdidaktische Herausforderung. In: Winkler, Iris; Masanek, Nicole \& Abraham, Ulf (Hrsg.): Poetisches Verstehen. Literaturdidaktische Positionen empirische Forschung - Projekte aus dem Deutschunterricht.

Baltmannsweiler: Schneider, 23-37 